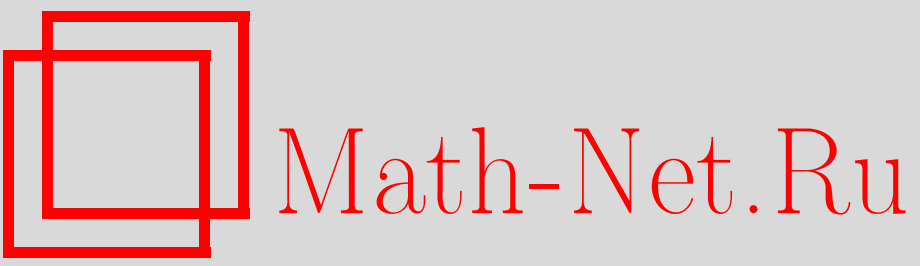

А. В. Разумов, В. И. Яснов, Гамильтонова редукция движения свободной частицы по группе $\mathrm{SL}(2, \mathbb{R}), T M \Phi, 1997$, том 110, номер 1, 149-161

DOI: https://doi.org/10.4213/tmf959

Использование Общероссийского математического портала Math-Net.Ru подразумевает, что вы прочитали и согласны с пользовательским соглашением

http://www.mathnet.ru/rus/agreement

Параметры загрузки:

IP: 52.23 .180 .231

26 апреля 2023 г., $13: 15: 23$ 


\author{
ТЕОРЕТИЧЕСКАЯ \\ И МАТЕМАТИЧЕСКАЯ \\ ФИЗИКА \\ Том 110, № 1 \\ январь, 1997
}

\author{
А.В. Разумов* , В.И. Яснов*
}

\title{
ГАМИЛЬТОНОВА РЕДУКЦИЯ ДВИЖЕНИЯ СВОБОДНОЙ ЧАСТИЦЫ ПО ГРУППЕ $\mathrm{SL}(2, \mathbb{R})$
}

Исследована структура приведенного фазового пространства, возникающего при гамильтоновой редукции фазового пространства, соответствующего свободному движению частицы по группе $\operatorname{SL}(2, \mathbb{R})$. Рассматриваемая редукция основывается на введении связей аналогичных тем, которые используются при редукции модели Весса-Зумино-Новикова-Виттена к тодовским системам. Показано, что приведенное фазовое пространство диффеоморфно либо объединению двух двумерных плоскостей, либо цилиндру $S^{1} \times \mathbb{R}$. Для обоих случаев построены канонические координаты и показано, что в первом случае приведенное фазовое пространство симплектоморфно объединению двух кокасательных расслоений $T^{*}(\mathbb{R})$, снабженных канонической симплектической структурой, а во втором случае оно симплектоморфно кокасательному расслоению $T^{*}\left(S^{1}\right)$, снова снабженному канонической симплектической структурой.

\section{1. ВВЕДЕНИЕ}

В настояшее время хорошо известен метод получения различных тодовских систем при помоши гамильтоновой редукции модели Весса-Зумино-Новикова-Виттена (ВЗНВ). В частности, модель ВЗНВ для группы Ли $\mathrm{SL}(2, \mathbb{R})$ приводит к простейшей тодовской системе - уравнению Лиувилля. Основным техническим приемом при проведении обсуждаемой редукции является использование разложения Гаусса группового элемента, описываюшего конфигурацию модели ВЗНВ (см., например, [1]). Уже в одной из первых работ по гамильтоновой редукции модели ВЗНВ [2] подчеркивалось, что разложение Гаусса является локальным. Следовательно, приведенное фазовое пространство совпадает с фазовым пространством соответствующей тодовской модели только локально и может иметь более сложную глобальную структуру. $\mathrm{K}$ сожалению, пока не удалось получить исчерпьвающую информацию о топологии приведенного фазового пространства для случая полной двумерной модели ВЗНВ.

В работе [3] была в этой связи рассмотрена одномерная модель, получаемая из модели ВЗНВ ограничением на конфигурации, не зависяшие от пространственной переменной. Фактически это модель, описываюшая движение частишы по соответствуюшему групповому многообразию. Авторы работы [3] показали, что для группы Ли $\mathrm{SL}(2, \mathbb{R})$ в зависимости от значений параметров, характеризуюших редукцию, имеются два различных случая. В первом случае приведенное фазовое пространство состоит из объе-

${ }^{*}$ Институт физики высоких энергий 
динения двух фазовых пространств одномерной модели Лиувилля. Во втором случае фазовое пространство имеет более сложную топологию. Детальное, хотя и далеко не полное, исследование приведенных фазовых пространств для различных групп Ли было проведено в [4].

Квантование редуцированной системы для случая группы $\operatorname{SL}(2, \mathbb{R})$ изучалось в работе [5]. В этой работе приведенное фазовое пространство рассматривалось как поверхность, склеенная из двух частей. Квантование проводилось для каждой части отдельно, а затем рассматривалась процедура склейки соответствующих волновых функций. Другой вариант квантования, в котором также используется локальное представление приведенного фазового пространства, представлен в [6].

На наш взгляд, наиболее убедительным явилось бы квантование, основанное на глобальном описании фазового пространства. В настояшей работе мы делаем первый шаг в этом направлении, показывая, что приведенное фазовое пространство модели, основанной на группе Ли $\mathrm{SL}(2, \mathbb{R})$, диффеоморфно либо объединению двух двумерных плоскостей, либо цилиндру $S^{1} \times \mathbb{R}$. При этом для обоих случаев мы строим канонические координаты и показываем, что в первом случае приведенное фазовое пространство симплектоморфно объединению двух кокасательных расслоений $T^{*}(\mathbb{R})$, снабженных канонической симплектической структурой, а во втором случае оно симплектоморфно кокасательному расслоению $T^{*}\left(S^{1}\right)$, снова снабженному канонической симплектической структурой. Заметим, что в работе [6] уже отмечалось, что приведенное фазовое пространство топологически имеет вьшеописанную структуру, однако дифференциально геометрическая эквивалентность доказана не была.

\section{2. МАТРИЧНЫЕ ГРУППЫ ЛИ}

Пусть $G$ есть действительная матричная группа Ли; другими словами, $G$ - некоторая подгруппа Ли группы Ли $\mathrm{GL}(m, \mathbb{R})$. Отождествим алгебру Ли $\mathfrak{g}$ группы Ли $G$ с соответствуюшей подалгеброй алгебры Ли $\mathfrak{g} l(m, \mathbb{R})$. Обозначим через $g$ матричнозначную функцию на $G$, заданную формулой

$$
g_{i j}(a)=a_{i j}
$$

для любого $a=\left\|a_{i j}\right\| \in G$.

Форма Маурера-Картана для случая матричной группы $G$ является матричнозначной 1-формой, заданной соотношением

$$
\theta=g^{-1} d g
$$

Форма $\theta$ инвариантна относительно правых сдвигов в группе $G$, принимает значения в алгебре Ли $\mathfrak{g}$ и удовлетворяет соотношению

$$
d \theta+\theta \wedge \theta=0 .
$$

Введем на $G$ локальные координаты $y^{\mu}$ и представим форму Маурера-Картанав виде

$$
\theta=\left(g^{-1} \partial_{\mu} g\right) d y^{\mu}
$$


где $\partial_{\mu}=\partial / \partial y^{\mu}$. Матричнозначные функции $g^{-1} \partial_{\mu} g$ принимают значения в алгебре Ли $\mathfrak{g}$. Выберем некоторый базис $e_{\alpha}$ в $\mathfrak{g}$. Структурные константы $c_{\alpha \beta}^{\gamma}$ алгебры Ли $\mathfrak{g}$ определяются из соотношений

$$
\left[e_{\alpha}, e_{\beta}\right]=c_{\alpha \beta}^{\gamma} e_{\gamma} .
$$

Разлагая $g^{-1} \partial_{\mu} g$ по базису $e_{\alpha}$, получаем

$$
\theta=e_{\alpha} \theta_{\mu}^{\alpha} d y^{\mu}
$$

Нетрудно убедиться в том, что соотношение (2.2) эквивалентно равенствам

$$
\partial_{\mu} \theta_{\nu}^{\alpha}-\partial_{\nu} \theta_{\mu}^{\alpha}+c_{\beta \gamma}^{\alpha} \theta_{\mu}^{\beta} \theta_{\nu}^{\gamma}=0
$$

Можно показать, что матрица $\left\|\theta_{\mu}^{\alpha}\right\|$ невырож дена. Обозначим матричные элементы обратной матришы через $\xi_{\alpha}^{\mu}$. Таким образом, имеем

$$
\xi_{\alpha}^{\mu} \theta_{\mu}^{\beta}=\delta_{\alpha}^{\beta} .
$$

Используя это соотношение и равенства $(2.5)$, получаем

$$
\xi_{\alpha}^{\nu} \partial_{\nu} \xi_{\beta}^{\mu}-\xi_{\beta}^{\nu} \partial_{\nu} \xi_{\alpha}^{\mu}-c_{\alpha \beta}^{\gamma} \xi_{\gamma}^{\mu}=0
$$

Существует также левоинвариантная форма Маурера-Картана $\bar{\theta}$, задаваемая формулой

$$
\bar{\theta}=d g g^{-1} .
$$

Форма $\bar{\theta}$ принимает значения в алгебре Ли $\mathfrak{g}$ и удовлетворяет соотношению

$$
d \bar{\theta}-\bar{\theta} \wedge \bar{\theta}=0 .
$$

Используя локальные координаты $y^{\mu}$ и базис $e_{\alpha}$, представим форму $\bar{\theta}$ в виде

$$
\bar{\theta}=\partial_{\mu} g g^{-1} d y^{\mu}=e_{\alpha} \bar{\theta}_{\mu}^{\alpha} d y^{\mu} .
$$

Из соотношения (2.8) следуют равенства

$$
\partial_{\mu} \bar{\theta}_{\nu}^{\alpha}-\partial_{\nu} \bar{\theta}_{\mu}^{\alpha}-c_{\beta \gamma}^{\alpha} \bar{\theta}_{\mu}^{\beta} \bar{\theta}_{\nu}^{\gamma}=0
$$

Матрица $\left\|\bar{\theta}_{\mu}^{\alpha}\right\|$ невырождена. Следовательно, существует матрица $\left\|\bar{\xi}_{\alpha}^{\mu}\right\|$, где функции $\bar{\xi}_{\alpha}^{\mu}$ определяются из соотношений

$$
\bar{\xi}_{\alpha}^{\mu} \bar{\theta}_{\mu}^{\beta}=\delta_{\alpha}^{\beta}
$$

Из равенств (2.10) получаем

$$
\bar{\xi}_{\alpha}^{\nu} \partial_{\nu} \bar{\xi}_{\beta}^{\mu}-\bar{\xi}_{\beta}^{\nu} \partial_{\nu} \bar{\xi}_{\alpha}^{\mu}+c_{\alpha \beta}^{\gamma} \bar{\xi}_{\gamma}^{\mu}=0
$$


Напомним, что присоединенное представление для случая матричной группы Ли описывается формулой

$$
\operatorname{Ad}(a) u=a u a^{-1}, \quad a \in G, \quad u \in \mathfrak{g} .
$$

Сравнивая определения (2.1) и (2.7) форм $\theta$ и $\bar{\theta}$, имеем

$$
\bar{\theta}=g \theta g^{-1}=\operatorname{Ad}(g) \circ \theta .
$$

Матричные элементы $\operatorname{Ad}_{\alpha}^{\beta}(a)$ присоединенного представления относительно базиса $e_{\alpha}$ определяются равенством

$$
\operatorname{Ad}(a) e_{\alpha}=a e_{\alpha} a^{-1}=e_{\beta} \operatorname{Ad}_{\alpha}^{\beta}(a)
$$

Используя это равенство и соотношения (2.4) и (2.9), получаем

$$
\bar{\theta}_{\mu}^{\alpha}=\operatorname{Ad}_{\beta}^{\alpha}(g) \theta_{\mu}^{\beta}
$$

что также может быть записано в виде

$$
\operatorname{Ad}_{\beta}^{\alpha}(g)=\bar{\theta}_{\mu}^{\alpha} \xi_{\beta}^{\mu}
$$

В заключение этого раздела получим систему дифференциальных уравнений, которым удовлетворяют матричные элементы присоединенного представления группы $G$. Из равенства (2.12) следует, что

$$
\partial_{\mu}\left(g e_{\beta} g^{-1}\right)=e_{\gamma} \partial_{\mu}\left(\operatorname{Ad}_{\beta}^{\gamma}(g)\right)
$$

Легко убедиться в том, что

$$
\partial_{\mu}\left(g e_{\beta} g^{-1}\right)=\left[\partial_{\mu} g g^{-1}, g e_{\beta} g^{-1}\right]
$$

Используя равенство

$$
\bar{\xi}_{\alpha}^{\mu} \partial_{\mu} g g^{-1}=e_{\alpha}
$$

которое следует из (2.9), имеем

$$
\bar{\xi}_{\alpha}^{\mu}\left(g e_{\beta} g^{-1}\right)=e_{\gamma} c_{\alpha \delta}^{\gamma} \operatorname{Ad}_{\beta}^{\delta}(g)
$$

Соотношение (2.14) теперь дает

$$
\bar{\xi}_{\alpha}^{\mu} \partial_{\mu}\left(\operatorname{Ad}_{\beta}^{\gamma}(g)\right)=c_{\alpha \delta}^{\gamma} \operatorname{Ad}_{\beta}^{\delta}(g)
$$

\section{2. СВОБОДНОЕ ДВИЖЕНИЕ ПО МАТРИЧНОЙ ГРУППЕ ЛИ}

Движение точечной частищы по матричной группе Ли $G$ описывается отображением $g(t)$, сопоставляюшим каждому моменту времени элемент группы $G$. Предположим, что скалярное произведение на $\mathfrak{g}$, заданное соотношением

$$
(u, v)=\operatorname{tr}(u v)
$$


для любых $u, v \in \mathfrak{g}$, является невырожденным. Это предположение эквивалентно допущению невырожденности матрицы $c=\left\|c_{\alpha \beta}\right\|$, где

$$
c_{\alpha \beta}=\operatorname{tr}\left(e_{\alpha} e_{\beta}\right) .
$$

Скалярное произведение (3.1) инвариантно относительно присоединенного действия группы $G$ в $\mathfrak{g}$, определяемого присоединенным представлением, что эквивалентно справедливости равенств

$$
\operatorname{Ad}_{\alpha}^{\gamma}(a) \operatorname{Ad}_{\beta}^{\delta}(a) c_{\gamma \delta}=c_{\alpha \beta} .
$$

Свободному движению по группе $G$ соответствует лагранжиан

$$
L=\frac{1}{2} \operatorname{tr}\left(g^{-1} \dot{g} g^{-1} \dot{g}\right),
$$

где точка означает производную по $t$. Лагранжиан (3.4) инвариантен относительно левых и правых сдвигов в группе $G$. Используя равенство $(2.4)$, имеем

$$
g^{-1} \dot{g}=\left(g^{-1} \partial_{\mu} g\right) \dot{y}^{\mu}=e_{\alpha} \theta_{\mu}^{\alpha} \dot{y}^{\mu},
$$

что позволяет нам переписать выражение для лагранжиана $L$ в терминах координат $y^{\mu}$ и скоростей $\dot{y}^{\mu}$ :

$$
L=\frac{1}{2} \dot{y}^{\mu} G_{\mu \nu} \dot{y}^{\nu}
$$

где

$$
G_{\mu \nu}=\theta_{\mu}^{\alpha} c_{\alpha \beta} \theta_{\nu}^{\beta}=\bar{\theta}_{\mu}^{\alpha} c_{\alpha \beta} \bar{\theta}_{\nu}^{\beta}
$$

являются компонентами биинвариантной метрики на $G$. Последнее равенство в $(3.5)$ следует из (3.3).

Рассмотрим теперь гамильтонову формулировку модели. Фазовое пространство в этом случае есть кокасательное расслоение $T^{*}(G)$, снабженное канонической симплектической структурой. Локальные координаты $y^{\mu}$ на $G$ порождают локальные канонические координаты $y^{\mu}, p_{\mu}$ на $T^{*}(G)$, и каноническая симплектическая 2-форма имеет в этих координатах вид

$$
\Omega=d\left(p_{\mu} d y^{\mu}\right) .
$$

Такая симплектическая форма приводит к следуюшим скобкам Пуассона для координатных функций $y^{\mu}$ и $p_{\mu}$ :

$$
\left\{y^{\mu}, y^{\nu}\right\}=0, \quad\left\{p_{\mu}, p_{\nu}\right\}=0, \quad\left\{y^{\mu}, p_{\nu}\right\}=\delta_{\nu}^{\mu} .
$$

Преобразование Лежандра описывается в рассматриваемом случае соотношениями

$$
p_{\mu}=\frac{\partial L}{\partial \dot{y}^{\mu}}=G_{\mu \nu} \dot{y}^{\mu},
$$

и мы имеем следуюшее выражение для гамильтониана системы:

$$
H=\frac{1}{2} p_{\mu} G^{\mu \nu} p_{\nu}
$$


где $G^{\mu \nu}$ - матричные элементы матрицы, обратной к матрице $\left\|G_{\mu \nu}\right\|$. Явное выражение для $G^{\mu \nu}$ имеет вид

$$
G^{\mu \nu}=\xi_{\alpha}^{\mu} c^{\alpha \beta} \xi_{\beta}^{\nu}=\bar{\xi}_{\alpha}^{\mu} c^{\alpha \beta} \bar{\xi}_{\beta}^{\nu},
$$

где $c^{\alpha \beta}$ - матричные элементы матрицы, обратной к матрице $\left\|c_{\alpha \beta}\right\|$.

Определим функции $j_{\alpha}=-\xi_{\alpha}^{\mu} p_{\mu}, \bar{\jmath}_{\alpha}=-\bar{\xi}_{\alpha}^{\mu} p_{\mu}$. Принимая во внимание $(2.13)$, получаем

$$
j_{\alpha}=\operatorname{Ad}_{\alpha}^{\beta}(g) \bar{\jmath}_{\beta}
$$

Из (2.6) и (2.11) вытекают следующие выражения для скобок Пуассона функций $j_{\alpha}$ и $\bar{\jmath}_{\alpha}$ :

$$
\left\{j_{\alpha}, j_{\beta}\right\}=c_{\alpha \beta}^{\gamma} j_{\gamma}, \quad\left\{\bar{\jmath}_{\alpha}, \bar{\jmath}_{\beta}\right\}=-c_{\alpha \beta}^{\gamma} \bar{\jmath}_{\gamma}
$$

в то время как уравнения (2.15) и соотношение (3.7) дают

$$
\left\{j_{\alpha}, \bar{\jmath}_{\beta}\right\}=0
$$

Функции $j_{\alpha}$ и $\bar{\jmath}_{\alpha}$ являются соответственно генераторами правых и левых сдвигов на группе $G$. Действительно, из соотношений (2.3) и (2.4) получаем

$$
\xi_{\alpha}^{\mu} \partial_{\mu} g=g e_{\alpha}
$$

Это равенство позволяет легко показать, что

$$
\left\{j_{\alpha}, g\right\}=g e_{\alpha}
$$

Аналогично, используя (2.9), получаем

$$
\left\{\bar{\jmath}_{\alpha}, g\right\}=e_{\alpha} g
$$

Как это следует из (3.6), гамильтониан системы в терминах функций $j_{\alpha}$ или $\bar{\jmath}_{\alpha}$ имеет следующий вид:

$$
H=\frac{1}{2} j_{\alpha} c^{\alpha \beta} j_{\alpha}=\frac{1}{2} \bar{\jmath}_{\alpha} c^{\alpha \beta} \bar{\jmath}_{\beta}
$$

Используя (2.4), получаем

$$
d y^{\mu}=\xi_{\alpha}^{\mu} c^{\alpha \beta} \operatorname{tr}\left(e_{\beta} \theta\right)
$$

Таким образом, справедливо равенство

$$
\Omega=-\operatorname{tr}(j \theta),
$$

где мы ввели обозначение

$$
j=j_{\alpha} c^{\alpha \beta} e_{\beta}
$$

Аналогично получаем

$$
\Omega=-\operatorname{tr}(\bar{\jmath} \bar{\theta}),
$$


где

$$
\bar{\jmath}=\bar{\jmath}_{\alpha} c^{\alpha \beta} e_{\beta} .
$$

Из формул (3.7) и (3.3) следует, что матричнозначные функции $j$ и $\bar{\jmath}$ связаны соотношением

$$
\bar{\jmath}=g j g^{-1}=\operatorname{Ad}(g) \circ j .
$$

\section{4. ГРУППА ЛИ $\mathrm{SL}(2, \mathbb{R})$}

Группа Ли $\mathrm{SL}(2, \mathbb{R})$ состоит из действительных $2 \times 2$-матриц

$$
a=\left(\begin{array}{ll}
a_{11} & a_{12} \\
a_{21} & a_{22}
\end{array}\right)
$$

удовлетворяюших соотношению

$$
\operatorname{det} a=a_{11} a_{22}-a_{12} a_{21}=1 \text {. }
$$

Алгебра Ли группы $\operatorname{SL}(2, \mathbb{R})$ есть алгебра Ли $\mathfrak{s l}(2, \mathbb{R})$, состоящая из действительных $2 \times 2$-матриц с нулевым следом. Выберем в $\mathfrak{s l}(2, \mathbb{R})$ канонический базис:

$$
e_{1}=x_{-}=\left(\begin{array}{ll}
0 & 0 \\
1 & 0
\end{array}\right), \quad e_{2}=h=\left(\begin{array}{cc}
1 & 0 \\
0 & -1
\end{array}\right), \quad e_{3}=x_{+}=\left(\begin{array}{ll}
0 & 1 \\
0 & 0
\end{array}\right)
$$

При таком выборе базиса в соответствии с (3.2) имеем

$$
\left\|c_{\alpha \beta}\right\|=\left(\begin{array}{ccc}
0 & 0 & 1 \\
0 & 2 & 0 \\
1 & 0 & 0
\end{array}\right), \quad\left\|c^{\alpha \beta}\right\|=\left(\begin{array}{ccc}
0 & 0 & 1 \\
0 & 1 / 2 & 0 \\
1 & 0 & 0
\end{array}\right)
$$

Вводя обозначения

$$
\begin{aligned}
& j_{-}=j_{1}, \quad j_{0}=j_{2}, \quad j_{+}=j_{3}, \\
& \bar{\jmath}_{-}=\bar{\jmath}_{1}, \quad \bar{\jmath}_{0}=\bar{\jmath}_{2}, \quad \bar{\jmath}_{+}=\bar{\jmath}_{3},
\end{aligned}
$$

для матричнозначных функций $j$ и $\bar{\jmath}$ получаем выражения

$$
j=\left(\begin{array}{cc}
j_{0} / 2 & j_{-} \\
j_{+} & -j_{0} / 2
\end{array}\right), \quad \bar{\jmath}=\left(\begin{array}{cc}
\bar{\jmath}_{0} / 2 & \bar{\jmath}_{-} \\
\bar{\jmath}_{+} & -\bar{\jmath}_{0} / 2
\end{array}\right) .
$$

Теперь, используя соотношение (3.10), имеем

$$
\begin{gathered}
\bar{\jmath}_{-}=\left(g_{11}\right)^{2} j_{-}-g_{11} g_{12} j_{0}-\left(g_{12}\right)^{2} j_{+} \\
\bar{\jmath}_{0}=-2 g_{11} g_{21} j_{-}+\left(g_{11} g_{22}+g_{12} g_{21}\right) j_{0}+2 g_{22} g_{12} j_{+}, \\
\bar{\jmath}_{+}=-\left(g_{21}\right)^{2} j_{-}+g_{22} g_{21} j_{0}+\left(g_{22}\right)^{2} j_{+}
\end{gathered}
$$


Для рассматриваемого случая группы Ли $\mathrm{SL}(2, \mathbb{R})$ справедливо следуюшее выражение для обратного элемента:

$$
a^{-1}=\left(\begin{array}{cc}
a_{22} & -a_{12} \\
-a_{21} & a_{11}
\end{array}\right)
$$

Отсюда для формы Маурера-Картана получаем

$$
\theta=\left(\begin{array}{cc}
g_{22} d g_{11}-g_{12} d g_{21} & g_{22} d g_{12}-g_{12} d g_{22} \\
-g_{12} d g_{11}+g_{11} d g_{12} & -g_{21} d g_{12}+g_{11} d g_{22}
\end{array}\right)
$$

Принимая теперь во внимание (3.9) и (4.2), для симплектической формы $\Omega$ имеем

$$
\begin{aligned}
\Omega= & d\left[j_{-}\left(g_{21} d g_{11}-g_{11} d g_{21}\right)+j_{0}\left(g_{11} d g_{22}-g_{22} d g_{11}+\right.\right. \\
& \left.\left.+g_{12} d g_{21}-g_{21} d g_{12}\right) / 2+j_{+}\left(g_{12} d g_{22}-g_{22} d g_{12}\right)\right]
\end{aligned}
$$

\section{5. РЕДУЦИРОВАННОЕ ФАЗОВОЕ ПРОСТРАНСТВО}

Интересуюшая нас редукция фазового пространства осуществляется посредством наложения двух связей первого рода

$$
j_{+}=\mu, \quad \bar{\jmath}_{-}=\nu
$$

где $\mu$ и $\nu$ - две действительные константы, отличные от нуля. Связи (5.1) порождают на определяемой ими поверхности действие двумерной группы Ли. Это действие свободно, что приводит к расслоению поверхности связей на двумерные орбиты. Как следует из общей теории гамильтоновой редукции, пространство орбит является симплектическим многообразием с симплектической структурой, наследуемой из полного фазового пространства. Это симплектическое многообразие обычно называют приведенным фазовым пространством. В рассматриваемом случае приведенное фазовое пространство может быть реализовано как поверхность, получаемая в результате пересечения поверхности связей с поверхностью, заданной соотношениями, называемыми калибровочными условиями или калибровками. Отметим, что проекция рассматриваемого действия на конфигурационное пространство не является свободным действием, поэтому калибровочные условия должны налагаться на координаты $j_{\alpha}$ и $\bar{\jmath}_{\alpha}$. Нетрудно убедиться в том, что допустимыми калибровочными условиями будут, например, соотношения

$$
j_{0}=0, \quad \bar{\jmath}_{0}=0
$$

Учитывая эти калибровочные условия, мы видим, что приведенное фазовое пространство задается уравнениями

$$
\begin{gathered}
\left(g_{11}\right)^{2} j_{-}-\left(g_{12}\right)^{2} \mu=\nu, \\
g_{22} g_{12} \mu-g_{11} g_{21} j_{-}=0, \\
g_{11} g_{22}-g_{12} g_{21}=1 .
\end{gathered}
$$


Умножая (5.3) на $g_{11}$ и учитывая (5.2) и (5.4), получаем

$$
g_{12} \mu-g_{21} \nu=0
$$

Подставляя это равенство в (5.3), приходим к равенству

$$
g_{12}\left(g_{22} \nu-g_{11} j_{-}\right)=0
$$

С другой стороны, принимая во внимание (5.5), мы можем переписать (5.2) в виде

$$
\left(g_{11}\right)^{2}-g_{12} g_{21} \nu=\nu
$$

что дает после учета (5.4) соотношение

$$
g_{11}\left(g_{22} \nu-g_{11} j_{-}\right)=0
$$

Так как функции $g_{12}$ и $g_{11}$ не могут принимать нулевое значение одновременно, то из (5.6) и (5.7) следует, что

$$
g_{22} \nu-g_{11} j_{-}=0 .
$$

Используя соотношения (5.5) и (5.8) для исключения функций $g_{21}$ и $g_{22}$, мы легко убедимся в том, что система уравнений (5.2)-(5.4) эквивалентна одному уравнению (5.2). Другими словами, приведенное фазовое пространство может рассматриваться как двумерная поверхность в трехмерном пространстве с координатами $g_{11}, g_{12}$ и $j_{-}$, заданная уравнением (5.2). Как это следует из (4.3), симплектическая форма на приведенном фазовом пространстве дается соотношением

$$
\Omega=\frac{\mu}{\nu} d\left[2 j_{-}\left(g_{12} d g_{11}-g_{11} d g_{12}\right)+g_{11} g_{12} d j_{-}\right] .
$$

Приведенное фазовое пространство имеет разную топологию в зависимости от величины параметров $\mu$ и $\nu$. В действительности имеются два принципиально различных варианта, определяемых относительным знаком этих параметров.

Предположим сначала, что параметры $\mu$ и $\nu$ имеют одинаковьй знак. Без потери обшности можно положить $\mu=\nu=1$. Из (5.2) следует, что в рассматриваемом случае $g_{11}$ не может обрашаться в нуль и координата $j_{-}$может быть выражена через $g_{11}$ и $g_{12}$ :

$$
j_{-}=\frac{\left(g_{12}\right)^{2}+1}{\left(g_{11}\right)^{2}} \text {. }
$$

Таким образом, приведенное фазовое пространство топологически является объединением двух непересекающихся двумерных плоскостей. Ясно также, что эти плоскости могут быть реализованы как открытые подмножества (соответствующие условиям $g_{11}>0$ и $\left.g_{11}<0\right)$ плоскости, описываемой координатами $g_{11}$ и $g_{12}$.

Используя (5.10), из (5.9) получаем следуюшее выражение для симплектической формы на приведенном фазовом пространстве:

$$
\Omega=-2 \frac{d g_{12} \wedge d g_{11}}{\left(g_{11}\right)^{2}} .
$$


Теперь нетрудно ввести канонические координаты, полагая, например, для полуплоскости $g_{11}>0$

$$
Q=\ln g_{11}, \quad P=-2 \frac{g_{12}}{g_{11}} .
$$

Из (3.8) и (4.1) заключаем, что гамильтониан приведенной системы совпадает с $j_{-}$. Принимая во внимание (5.10) и равенства

$$
g_{11}=\exp Q, \quad g_{12}=-\frac{1}{2} P \exp Q
$$

получаем

$$
H=\frac{1}{4} P^{2}+\exp (-2 Q)
$$

Решения уравнений движения для системы с таким гамильтонианом хорошо известны, и мы их приводить не будем.

Рассмотрим теперь случай, когда параметры $\mu$ и $\nu$ имеют разные знаки, и положим $\mu=-\nu=-1$. Введем полярные координаты для $g_{11}$ и $g_{12}$ :

$$
g_{11}=R \sin \Phi, \quad g_{12}=R \cos \Phi
$$

Из уравнения (5.2) следует, что

$$
R^{2}=\frac{1}{j_{-} \sin ^{2} \Phi+\cos ^{2} \Phi}
$$

Мы можем рассматривать $\Phi$ и $j_{-}$как координаты на приведенном фазовом пространстве. Легко видеть, что эти координаты принимают только такие значения, для которых

$$
j_{-} \sin ^{2} \Phi+\cos ^{2} \Phi>0
$$

Симплектическая форма в терминах координат $\Phi$ и $j_{-}$имеет вид

$$
\Omega=-\frac{1}{j_{-} \sin ^{2} \Phi+\cos ^{2} \Phi} d j_{-} \wedge d \Phi .
$$

Таким образом, мы видим, что координаты $\Phi$ и $j_{-}$обладают двумя существенными недостатками. Во-первых, эти координаты не принимают произвольных значений, а, во-вторых, они не являются каноническими. Нетрудно убедиться в том, что обший вид координаты, сопряженной к $\Phi$, дается соотношением

$$
\Pi=-\frac{1}{\sin ^{2} \Phi} \ln \left(j-\sin ^{2} \Phi+\cos ^{2} \Phi\right)+F(\Phi),
$$

где $F(\Phi)$ - произвольная периодическая функция. Выбирая $F(\Phi)=0$, приходим к удобным каноническим координатам $\Phi$ и П. Заметим, что эти координаты принимают уже произвольные значения. 
Теперь легко показать, что $g_{11}, g_{12}$ и $j_{-}$выражаются через координаты $\Phi$ и П следующим образом:

$$
\begin{gathered}
g_{11}=\exp \left(\Pi \sin ^{2} \Phi / 2\right) \sin \Phi \\
g_{12}=\exp \left(\Pi \sin ^{2} \Phi / 2\right) \cos \Phi \\
j_{-}=\left(\exp \left(-\Pi \sin ^{2} \Phi\right)-\cos ^{2} \Phi\right) / \sin ^{2} \Phi .
\end{gathered}
$$

Соотношения (5.11)-(5.13) дают параметрическое представление приведенного фазового пространства, рассматриваемого как поверхность, заданная уравнением (5.2). При этом функции, входяшие в эти соотношения, являются бесконечно дифференцируемыми. Более того, соответствуюшие касательные векторы линейно независимы. Следовательно, приведенное фазовое пространство диффеоморфно цилиндру $S^{1} \times \mathbb{R}$. Учитьвая тот факт, что $\Phi$ и П суть канонические координаты, мы видим, что приведенное фазовое пространство симплектоморфно кокасательному расслоению $T^{*}\left(S^{1}\right)$, снабженному канонической симплектической структурой.

Гамильтониан системы дается в координатах $\Phi$ и П формулой

$$
H=\frac{1}{\sin ^{2} \Phi}\left(\cos ^{2} \Phi-\exp \left(-\Pi \sin ^{2} \Phi\right)\right) .
$$

Следовательно, гамильтоновы уравнения движения имеют вид

$$
\begin{gathered}
\dot{\Phi}=\exp \left(-\Pi \sin ^{2} \Phi\right) \\
\dot{\Pi}=2 \operatorname{ctg} \Phi\left(\frac{1}{\sin ^{2} \Phi}-\exp \left(-\Pi \sin ^{2} \Phi\right)\left(\Pi+\frac{1}{\sin ^{2} \Phi}\right)\right) .
\end{gathered}
$$

Несмотря на то что эти уравнения выглядят довольно сложно, они легко решаются. Действительно, гамильтониан системы есть сохраняюшаяся величина, имеюшая смысл энергии. Будем искать решение уравнений движения, для которого $H=\epsilon$. Используя (5.13) и (5.14), приходим к соотношению

$$
\epsilon=\frac{1}{\sin ^{2} \Phi}\left(\cos ^{2} \Phi-\dot{\Phi}\right)
$$

Рассмотрим функцию $T=\operatorname{tg} \Phi$. Для этой функции получаем уравнение

$$
\dot{T}=1-\epsilon T^{2} \text {. }
$$

В случае, если $\epsilon<0$, это уравнение имеет решение

$$
T(t)=\frac{1}{\sqrt{-\epsilon}} \tan (\sqrt{-\epsilon}(t-c)),
$$


где $c$ - постоянная интегрирования. Отсюда легко получаем

$$
\begin{gathered}
\cos 2 \Phi(t)=\frac{\epsilon+\tan ^{2}(\sqrt{-\epsilon}(t-c))}{\epsilon-\tan ^{2}(\sqrt{-\epsilon}(t-c))}, \\
\sin 2 \Phi(t)=-\frac{2 \sqrt{-\epsilon} \tan (\sqrt{-\epsilon}(t-c))}{\epsilon-\tan ^{2}(\sqrt{-\epsilon}(t-c))}, \\
\Pi(t)=\frac{\tan ^{2}(\sqrt{-\epsilon}(t-c))-\epsilon}{\tan ^{2}(\sqrt{-\epsilon}(t-c))} \ln \left[\frac{\epsilon+\epsilon \tan ^{2}(\sqrt{-\epsilon}(t-c))}{\epsilon-\tan ^{2}(\sqrt{-\epsilon}(t-c))}\right] .
\end{gathered}
$$

При $\epsilon>0$ уравнение (5.16) имеет решение

$$
T(t)=\frac{1}{\sqrt{\epsilon}} \tanh (\sqrt{\epsilon}(t-c))
$$

и мы приходим к соотношениям

$$
\begin{gathered}
\cos 2 \Phi(t)=\frac{\epsilon-\tanh ^{2}(\sqrt{\epsilon}(t-c))}{\epsilon+\tanh ^{2}(\sqrt{\epsilon}(t-c))}, \\
\sin 2 \Phi(t)=\frac{2 \sqrt{\epsilon} \tanh (\sqrt{\epsilon}(t-c))}{\epsilon+\tanh ^{2}(\sqrt{\epsilon}(t-c))} \\
\Pi(t)=-\frac{\tanh ^{2}(\sqrt{\epsilon}(t-c))+\epsilon}{\tanh ^{2}(\sqrt{\epsilon}(t-c))} \ln \left[\frac{\epsilon-\epsilon \tanh ^{2}(\sqrt{\epsilon}(t-c))}{\epsilon+\tanh ^{2}(\sqrt{\epsilon}(t-c))}\right] .
\end{gathered}
$$

Таким образом, мы имеем явное решение уравнений движения.

Для построения квантовой теории необходимо решить проблему упорядочения для гамильтониана. В любом случае в представлении, в котором пространство состояний реализуется как гильбертово пространство квадратично интегрируемых функций на окружности, получаемый оператор не будет локальным. Однако наличие явного решения классических уравнений движения позволяет надеяться, что задача на собственные значения гамильтониана может быть решена.

\section{6. ЗАКЛЮЧЕНИЕ}

В настояшей работе мы провели детальное исследование структуры приведенного фазового пространства, возникаюшего в результате гамильтоновой редукции фазового пространства, соответствуюшего свободному движению частищы по группе Ли $\mathrm{SL}(2, \mathbb{C})$. Мы показали, что приведенное фазовое пространство диффеоморфно либо объединению двух кокасательных расслоений $T^{*}(\mathbb{R})$, либо кокасательному расслоению $T^{*}\left(S^{1}\right)$. Более того, мы построили для обоих случаев канонические координаты и показали, что возникаюшие приведенные фазовые пространства симплектоморфны соответствуюшим кокасательным расслоениям, снабженным канонической симплектической структурой.

Отметим, что во втором из рассмотренных случаев гамильтониан системы имеет довольно сложную структуру. Однако классические уравнения движения могут быть проинтегрированы в явном виде, что позволяет надеяться на сушествование явного решения задачи квантования.

Авторы глубоко благодарны Г. Л. Рчеулишвили, Г. П. Пронько и М. В. Савельеву за интересные и плодотворные обсуждения. Настоящая работа выполнена при частичной поддержке Российского фонда фундаментальных исследований (проект № 95-01-00125а). 


\section{Список литературы}

[1] L. Fehér, L. O’Raifeartaigh, P. Ruelle, I. Tsutsui, A. Wipf. Phys. Rep. 1992. V. 222. № 1. P. 1.

[2] P. Forgács, A. Wipf, J. Balog, L. Fehér, L. O’Raifeartaigh. Phys. Lett. 1989. V. 227B. № 2. P. 214.

[3] I. Tsutsui, L. Fehér. Pror. Theor. Phys. Suppl. 1995. V. 118. № 1. P. 173.

[4] L. Fehér, I. Tsutsui. Regularization of Toda lattices by Hamiltonian reduction: Preprint INS-1123. Tokyo: Institute for Nuclear Study, University of Tokyo, 1995.

[5] T. Fülöp. Reduced SL $(2, \mathbb{R})$ WZNW quantum mechanics: Report 509. Budapest: Institute for Theoretical Physics, Eötvös University, 1995.

[6] H. Kobayashi, I. Tsutsui. Quantum mechanical Liouville model with attractive potential: Preprint INS-1124. Tokyo: Institute for Nuclear Study, University of Tokyo, 1996.

Поступила в редакцию 29.V.1996 г.

\section{A. V. Razumov, V.I. Yasnov \\ HAMILTONIAN REDUCTION OF FREE PARTICLE MOTION ON GROUP $\operatorname{SL}(2, \mathbb{R})$}

The structure of the reduced phase space arising in the Hamiltonian reduction of the phase space corresponding to a free particle motion on the group $\operatorname{SL}(2, \mathbb{R})$ is investigated. In the considered reduction the constraints similar to those in the Hamiltonian reduction of the Wess-Zumino-Novikov-Witten model to Toda systems are used. It is shown that the reduced phase space is diffeomorphic either to the union of two two-dimensional planes, or to the cylinder $S^{1} \times \mathbb{R}$. Canonical coordinates are constructed in both cases. In the first case the reduced phase space is sympectomorphic to the union of two cotangent bundles $T^{*}(\mathbb{R})$ endowed with the canonical symplectic structure, while in the second case it is symplectomorphic to the cotangent bundle $T^{*}\left(S^{1}\right)$ also endowed with the canonical sympectic structure. 